\title{
Pyroelectric Ceramics for Infrared Detection Applications
}

\author{
Padmaja Guggilla, A. K. Batra, J. R. Currie ${ }^{+}$, M. D. Aggarwal, B. Penn, R. B. Lal ${ }^{*}$ \\ Department of Physics, Alabama A\&M University \\ Normal, AL 35762 (USA) \\ ${ }^{+}$Avionics Department \\ NASA / Marshal Space Flight Center, Huntsville, AL 35812
}

\begin{abstract}
Pyroelectric and dielectric properties of modified PZT and PZ have been studied for their use in infrared detectors. Various material figure-of-merits for their use in infrared detector are calculated including when the pyroelectric element is placed on heat-sink condition. The results are compared with exiting candidate materials.
\end{abstract}

\section{Introduction}

Pyroelectric Infrared detectors offer the advantage of room temperature operation and wide spectral response In the past decades or so major thrust for infrared detection and the thermal imaging has been for military night vision, target acquisition and missile guidance. Non-military applications include infrared detecting head in imaging system for product inspection for faults isolation and quality assurance, automotive vision enhancement, fire detection and prevention, remote temperature measurements and medical diagnosis ( cancer detection and rheumatism), and monitoring burns patients. For all these applications, efforts have been made to develop uncooled infrared and imaging devices. Because cooled photon detector technologies require bulky and expensive cryogenics. The result of the efforts has been the use of crystalline ferroelectrics, such as triglycine sulfate (TGS), SBN; Lithium tantalate, and

\footnotetext{
* NASA Administrator's Fellow, Exploration Science and Technology Division XD40, NASA/Marshall Space Flight Center, AL 35812
} 
pyroelectric ceramics [1-6].

Ferroelectric oxide ceramics (PZT) for use in pyroelectric infrared detection offer a number of advantages over the the single sulfate, lithium tantalate, PVDF etc. They are relatively cheap to manufacture in large areas using standard mixed-ceramic oxide processes, are both mechanically and chemically robust. Furthermore, they are not hygroscopic as ate high performance TGS crystal elements. They also have high Curie temperature so that there is no danger of depoling during normal use. Their properties can be readily modified by inclusion of selected dopant elements into lattice, thereby controlling important parameters such as pyroelectric coefficient $(\mathrm{p}) \stackrel{+}{5}$ and dielectric constants $\left(\varepsilon^{\prime}, \varepsilon^{\prime \prime}\right)$ which have direct affect on

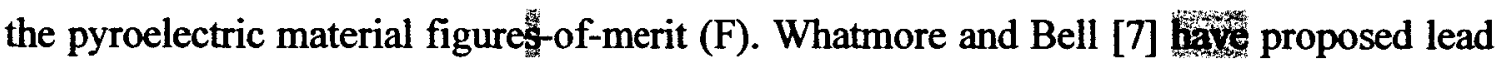
iron niobate lead titanate ceramics modified by the addition of uranium ceramics. The properties of modified lead zirconate ceramics and other materials are listed in table 1 [89]. Nevertheless, there remains immense interest in the improvement of quality and performance of these materials as requirements for future applications, in particular for military and medical applications.

In this communication, we present the results on investigation of lanthanummodified lead zirconate titanate (PLZT), abbreviated as STPZT-1 and doped PLZT with $\mathrm{MnO}_{2}$, abbreviated as STPZT-2, and commercially available modified lead zirconate ceramic (BM740) from Sensor Technology, Canada [10]. Their usefulness in pyroelectric infrared detectors is compared with results of other candidate pyroelectric materials. BM 740 is chosen as it exhibits low frequency drift over normal working temperature and the aging of permittivity is low [10]. 


\section{Experimental}

Ceramic samples were prepared using processes shown in Fig.1 by propriety fabrication technology of Sensor Technology, Canada [8]. The samples for electrical measurements were made in the form of disc $\$ 3.75 \mathrm{~mm}$ in diameter and $0.09 \mathrm{~mm}$ in thickness. Silver electrodes were screen printed on both sides of the samplest and then they were poled with ap appropriate field using the technique perfected by Sensor Technology, Canada. The dielectric permittivity were calculated by measuring capacitance at frequency of $1 \mathrm{kHz}$ at an applied signal field of $10 \mathrm{~V} / \mathrm{cm}$ using a QuadTech 1920 LCR meter in a three terminal cell designed in our laboratory. The pyroelectric current was measured using the Byer and Roundy method [9] and pyroelectric coefficient (p) was calculated using a relationship:

$$
p=\left(\frac{I}{A}\right) /\left(\frac{d T}{d t}\right),
$$

where $I$ is the pyroelectric current, $A$ is the area of electrodes, $\frac{d T}{d t}$ is the rate of change of temperature. The current $(I)$ is measured using a 6517 Keithley electrometer and temperature is measured by an HP34970 digital multimeter. The measurements were

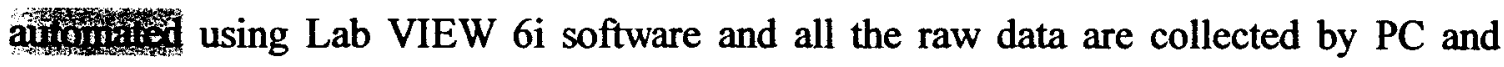
stored in a file. The schematic of complete set-up is shown in Fig. 2 and details are described elsewhere $[10]^{5}$

The-Using the above cited electrical parameters, the following material figureof-merit (F) [7] for assessing the characteristics of single element pyroelectric detector, operating in optimum manner, were calculated for comparison, assuming the volume 
specific heat from the literature, $F_{\text {sink }}$ is the figure-of-merit when the pyroelectric element is attached on the heat sink [10].

$F_{I}=p / c \quad$ for high current responsivity, $\quad F_{\mathrm{I}, \text { sink }}=p / k$,

$\mathrm{F}_{v}=p / \mathrm{c} \varepsilon^{\prime} \quad$ for high voltage responsivity, $\quad \mathrm{F}_{v, \text { sink }}=\mathrm{p} / \varepsilon^{\prime} k$,

$\mathrm{F}_{\mathrm{D}}=p / \mathrm{c} \sqrt{\varepsilon^{\prime \prime}} \quad$ for high detectivity, $\quad$ and $\quad \mathrm{F}_{\mathrm{D}, \text { sink }}=\mathrm{p} / k \sqrt{ } \varepsilon^{\prime \prime}$,

where $p, \mathrm{c}, \mathrm{k}, \varepsilon^{\prime}$ and $\varepsilon^{\prime \prime}$ are the pyroelectric coefficient, the volume specific heat, the thermal conductivity, and the real and imaginary dielectric constants respectively.

\section{Results and Discussion}

Fig. 3 shows the variation of pyroelectric coefficient and dielectric constants with temperature respectively. These parameters increase with temperature which is typical of ferroelectrics. Table 1 lists the values obtained for dielectric constant and loss, pyroelectric coefficient and calculated material figures-of-merits (F) for STPZT-1, STPZT-2 and BM 740 ceramic samples at $50^{\circ} \mathrm{C}$ along with published data of other important materials for comparison. For calculation of figures-of-merit of samples fabricated and investigated in the present work, the default values of volume specific heat $\left(c^{\prime}\right)$ and thermal conductivity (k) are chosen from the literature. The values obtained for our STPZT-1 (PLZT) sample is lower than reported value as listed in table 1 [9], which is of importance in figures-of-merit for the high detectivity operation of values obtained for all the figures-of-merit for STPZT-2 (PLZT $\left.+\mathrm{MnO}_{2}\right)$ is higher than STPZT-1 due to lower dielectric constants and higher pyroelectric coefficient values

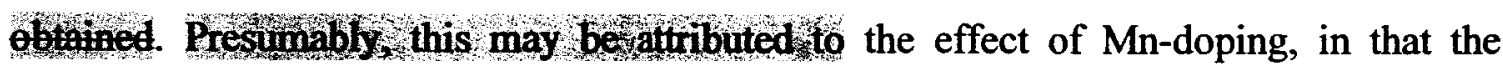
recharging $\left(\mathrm{Mn}^{4+} \mathrm{Mn}^{3+} \mathrm{Mn}^{2+}\right.$ ) of $\mathrm{Mn}$ ions partially substituted at the $\mathrm{B}$-site ( for $\mathrm{Zr}^{4+}$ or $\mathrm{Ti}^{4+}$ ions ) compensates for excessive charge of $\mathrm{La}^{3+}$ to the $\mathrm{A}$-site $\left(\mathrm{Pb}^{2+}\right.$ ions) caused by 
La-substitutions in the A-sites as has been reported by Glinchuk et al., [13]. This process results in a high resistivity and low loss which is important for IR sensors. In ceramic oxide ferroelectrics, the value of dielectric constants and pyroelectric coefficients depend on grain size,

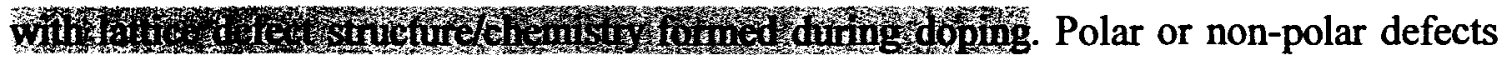
are formed in the crystal cell due to doping, direction of polarity of defect formed, and on the dipole interaction of the impurity defects and the host. The reduction in dielectric constant and loss is due to low frequency polarization. This may be because once the oxygen vacancies are locked into defect dipole pairs; they are much more immobile in STPZT-2 than STPZT-1. As can be seen from Fig.3, properties do not change greatly up to $100^{\circ} \mathrm{C}$, thus their performance is tof degraded with change in ambient temperatures 19. The propriethy modified PZ, BM740 which has high figures-of-merit for detectivity due to low loss and dielectric constant is also attractive for use in IR sensors.

\section{Conclusions}

Pyroelectric properties of various ceramics such as PLZT, PLZT $+\mathrm{MnO}_{2}$ and modified PZ for their applications to IR sensor materials are studied and compared with other candidate materials. The materials figlitesof of netil are calculated from measured parameters (dielectric constants and pyroelectric coefficient) for their use in pyroelectric infrared detectors. From the viewpoint of pyro sensor applications, our investigated ceramics PLZT (STPZT-1) PLZT $+\mathrm{MnO}_{2}(\mathrm{STPZT}-2)$ and modified PZ (BM 740) are remarkably attractive due to large pyroelectric coefficient and low dielectric constant and loss. It is worthy to mention that the materials investigated are also capable of high 
temperature applications. However, their actual use in infrared detector shall determine if proposed enhancement can be realized in practice. Further work is in progress to use these materials in thick films fabrication so that there performance be assessed for H.

\section{Acknowledgements}

This work is supported by US Army SMDC grant \# DASG60-03-1-0003. The authors would like to sincerely thank Mr. G. Sharp for fabrication of sample holder for capacitance measurements. Thanks to Drs. D. Waechter and E. Prasad of SensorTech, for their cooperation and suggestions. One of us (AKB) would like to thank Mrs. Mary Strong for her kind cooperation.

\section{References}

[1]. R. B. Lal, and A. K. Batra, Ferroelectrics 142, 51 (1993).

[2]. Jiann-Min. Chang, A. K. Batra, R. B. Lal, Crystal Growth \& Design, 7, (2002)

[3]. M. Banan, R. B. Lal, A. K. Batra, J. Mat. Sci. 27, 2291 (1992).

[4]. R. W. Whatmore, Rep. Prog. Phys., 49, 1335 (1986).

[5]. A. Rogalski, Prog. Quant. Elect, 27, 59 (2003).

[6]. H. P. Beerman, Infrared Physics 35, 115 (1975).

[7]. R. W. Whatmore and A. J. Bell, Ferroelectrics 142, 51 (1981).

[8]. Sidney B. Lal and Dilip K. Das-Gupta, Ferroelectrics Review 2(3-4), 217 (2000)

[9]. A. Lozinski, F. Wang, A. Uusimaki and S. Leppavuori Meas. Sci. Technol., 8, 33 (1997)

[10] www.sensortech.ca 
[11]. R. L. Byer and C. B. Roundy, Ferroelectrics, 3, 333 (1972)

[12] James R. Curie, MS thesis (2004) Alabama A\&M University, AL.

[13] M. D. Glinchuk, I. P. Bykov, V. Skorokhod and T. Kala, Ferroelectrics, 131, 233

(1992)

[14] A. Pelaiz Barranco, F. Calderon Pinar and O. Perez Martinez, Phys. Stat. Sol

(a), !86(3), 478(2001.

[15] 


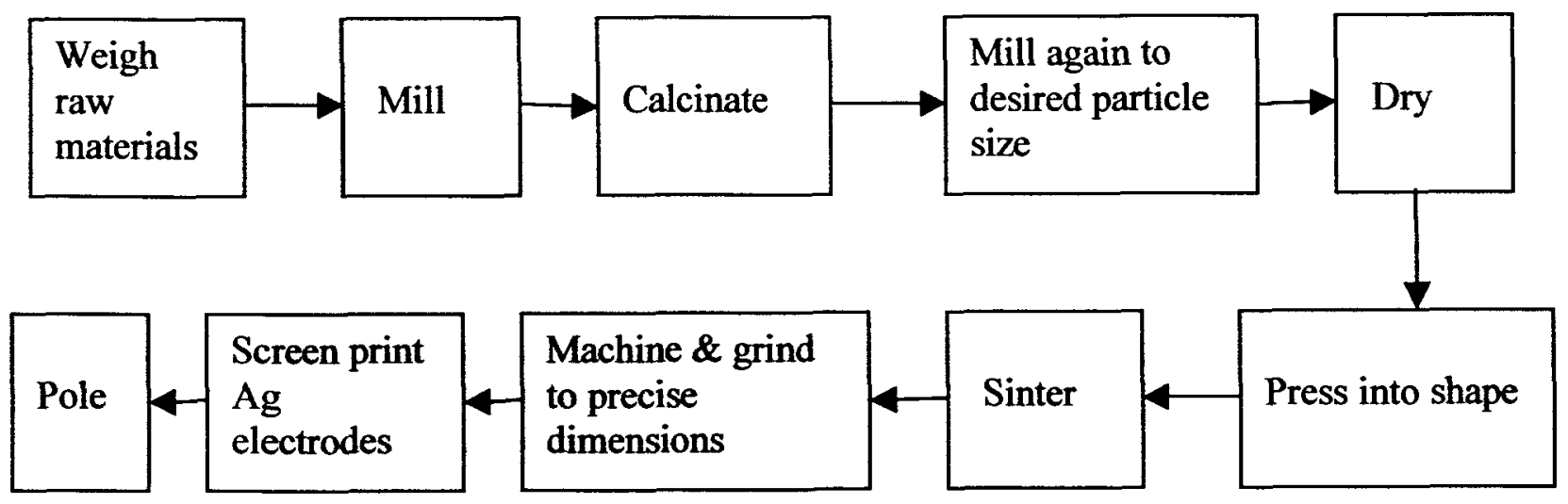

Fig. 1 Flow chart of sample preparation 


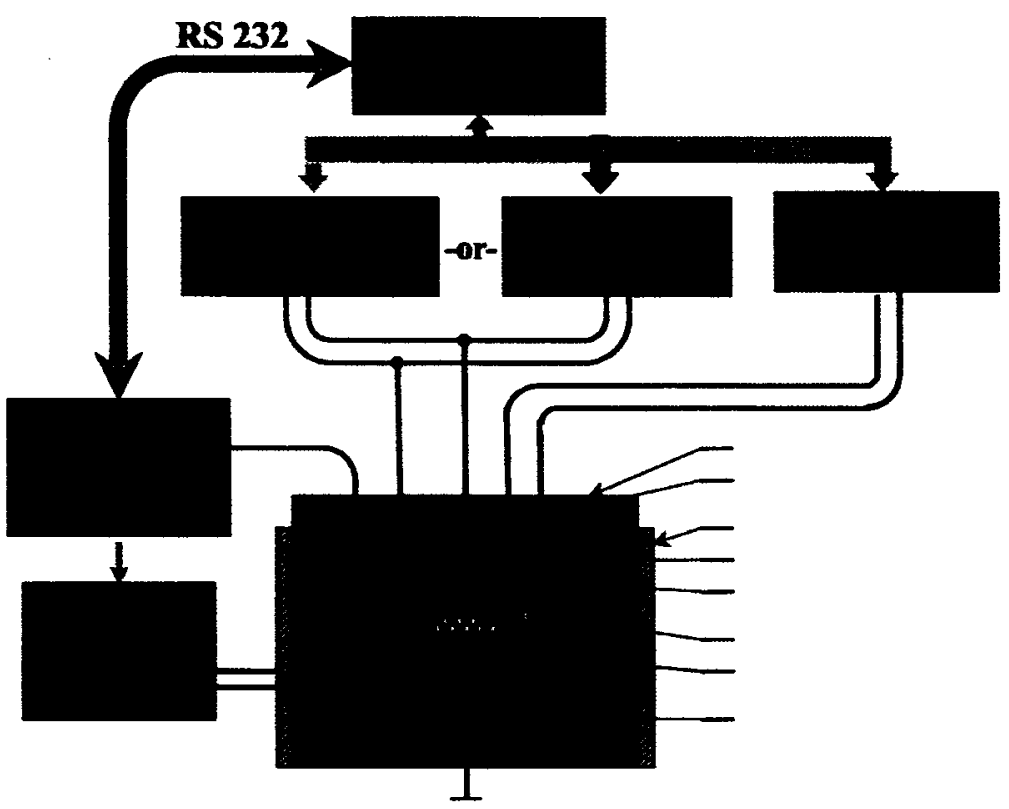

Fig. 2 Block Diagram of the pyroelectric and dielectric response measurement setup 
STPZT1
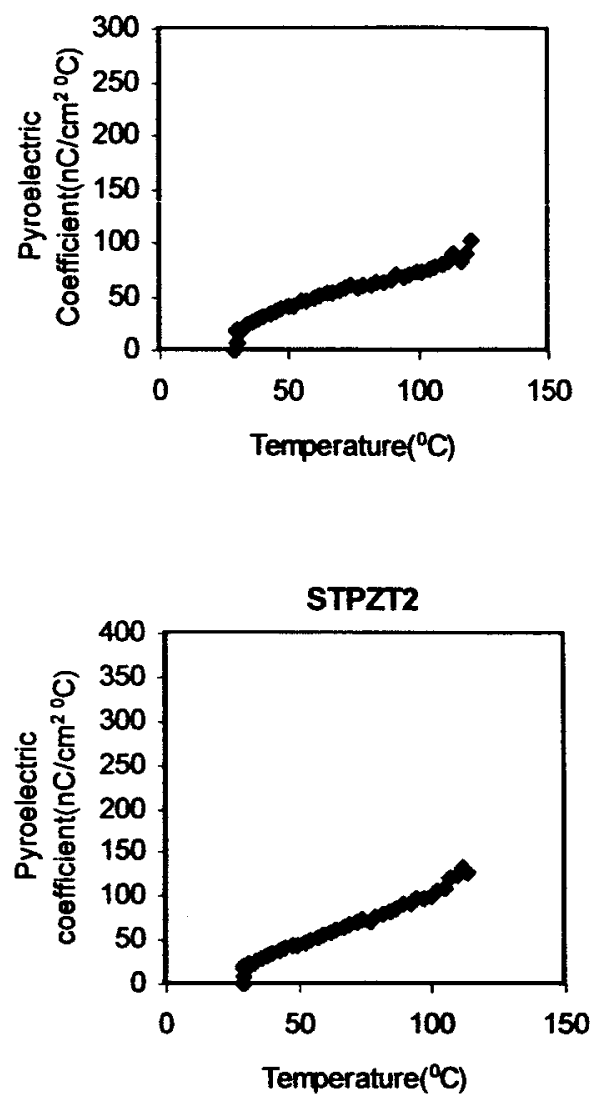

BM740

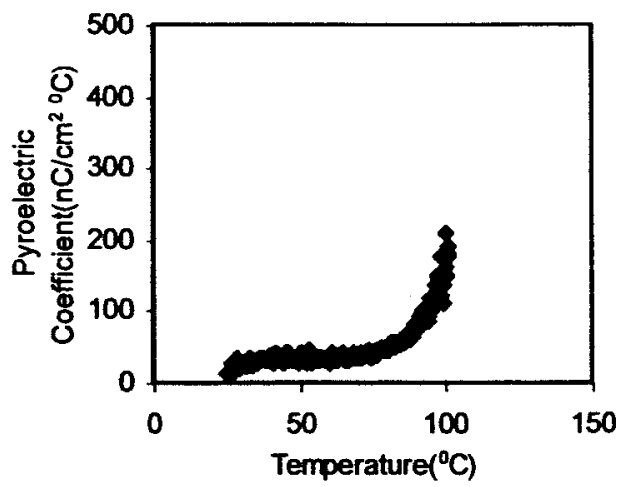

STPZT1

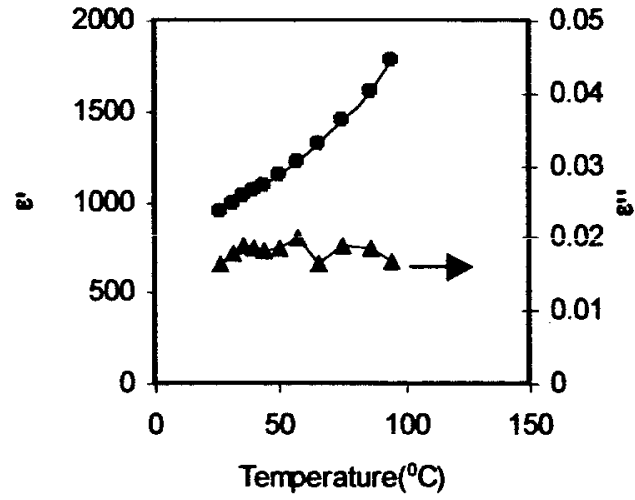

STPZT2

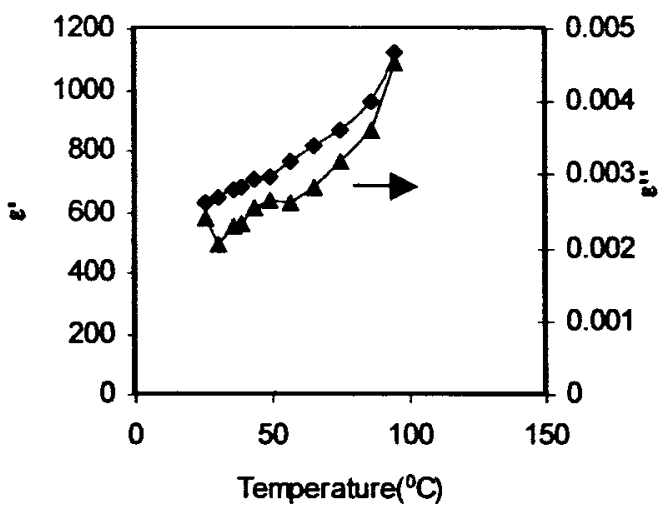

BM740

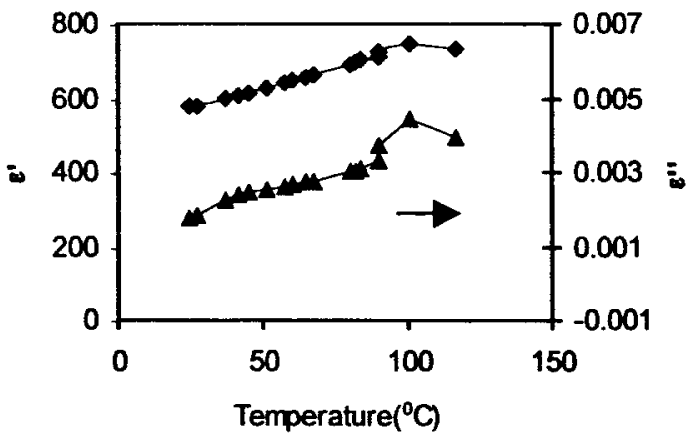

Fig. 3 The variation of pyroelectric coefficient and dielectric constants $\left(\varepsilon^{\prime}, \varepsilon^{\prime \prime}\right)$ with temperature 
Table 1 Physical properties and figures-of-merit of various pyroelectric materials

\begin{tabular}{|c|c|c|c|c|c|c|c|c|c|}
\hline Parameter & Units & $\begin{array}{l}\mathrm{LiTaO}_{3} \\
\text { (Crystal) }\end{array}$ & $\underset{\text { (Crystal) }}{\text { TGS }}$ & $\begin{array}{l}\text { Mod. PZT } \\
\text { (Ceramic) }\end{array}$ & $\begin{array}{c}\mathrm{PbTiO}_{3} \\
\text { (Ceramic) }\end{array}$ & $\begin{array}{l}\text { PLZT [9] } \\
\text { (Ceramic) }\end{array}$ & $\begin{array}{c}\text { STPZT1 } \\
\text { (Present } \\
\text { Work) }\end{array}$ & $\begin{array}{c}\text { STPZT2 } \\
\text { (Present } \\
\text { Work) }\end{array}$ & $\begin{array}{c}\text { BM740 } \\
\text { (Present } \\
\text { Work) }\end{array}$ \\
\hline$P$ & $\mu \mathrm{Cm}^{-2} \mathrm{~K}^{-1}$ & 180 & 280 & 380 & 250 & 350 & 379.5 & 408.5 & 300 \\
\hline$\varepsilon^{\prime}$ & - & 47 & 38 & 290 & 190 & 1129 & 1156.675 & 768.656 & 631.39 \\
\hline $\tan \delta$ & - & 0.005 & 0.01 & 0.003 & 0.006 & 0.028 & 0.01866 & 0.002639 & 0.0026 \\
\hline$\varepsilon^{\prime \prime}$ & - & 0.235 & 0.38 & 0.87 & 1.14 & 31.612 & 21.5835 & 2.0285 & 9.4096 \\
\hline$c^{\prime}$ & $10^{6} \mathrm{Jm}^{-3} \mathrm{~K}^{-1}$ & 3.2 & 2.3 & 2.5 & 3.2 & 2.5 & 2.5 & 2.5 & 3.2 \\
\hline$k$ & $\mathbf{W} \mathbf{m}^{-1} \mathbf{K}^{-1}$ & 3.9 & 0.65 & 0.8 & 1.76 & 0.8 & 0.8 & 0.8 & 1.76 \\
\hline$T_{\mathrm{C}}$ & ${ }^{\circ} \mathrm{C}$ & 620 & 49 & 200 & 490 & - & - & - & - \\
\hline $\mathbf{F}_{\mathbf{v}}$ & $10^{-6} \mu \mathrm{CJ}^{-1} \mathrm{~m}$ & 1.19 & 3.2 & 0.524 & 0.411 & 0.124 & 0.131 & 0.531 & 0.15 \\
\hline$F_{1}$ & $10^{-6} \mu \mathrm{CJ}^{-1} \mathrm{~m}$ & 56.25 & 121.74 & 152 & 78.13 & 140 & 151.8 & 163.4 & 93.75 \\
\hline$F_{D}$ & $10^{-6} \mu \mathrm{CJ}^{-1} \mathrm{~m}$ & 116.05 & 197.5 & 162.96 & 73.18 & 24.9 & 32.675 & 114.72 & 73.24 \\
\hline$F_{\mathrm{v}, \text { sink }}$ & $\mu \mathrm{Cm}^{-1} \mathrm{~W}^{-1}$ & 0.982 & 11.3 & 1.638 & 0.747 & 0.388 & 0.409 & 1.659 & 0.269 \\
\hline$F_{L, \text { sink }}$ & $\mu \mathrm{Cm}^{-1} \mathrm{~W}^{-1}$ & 46.15 & 430.77 & 475 & 142.05 & 437.5 & 474.38 & 510.625 & 170.45 \\
\hline$F_{D, \text { sink }}$ & $\mu \mathrm{Cm}^{-1} \mathrm{~W}^{-1}$ & 95.2 & 235 & 509.25 & 107.07 & 77.81 & 102.109 & 358.5 & 133.16 \\
\hline
\end{tabular}

